\title{
Geomagnetic effects of high-density plasma with southward magnetic field in the interplanetary coronal mass ejection observed on May 2-3, 1998
}

\author{
Haruka Adachi ${ }^{1}$, Tohru Sakurai ${ }^{1}$, and Katsuhide Marubashi ${ }^{2}$ \\ ${ }^{1}$ Department of Aeronautics and Astronautics, School of Engineering, Tokai University, 1117 Kitakaname, \\ Hiratsuka, Kanagawa 259-1292, Japan \\ ${ }^{2}$ 660-73 Mizuno, Sayama, Saitama 350-1317, Japan
}

(Received February 1, 2005; Revised June 10, 2005; Accepted September 5, 2005; Online published March 10, 2006)

\begin{abstract}
This paper aims to clarify the effect of high-density plasma in interplanetary coronal mass ejection (ICME) observed during the May 2-3, 1998 geomagnetic storm. The examination is performed based on the estimation of Dst index, which is calculated with the observed solar wind parameters of the ICME. The estimated Dst index variation is compared with Dst index variation provided by the World Data Center for Geomagnetism, Kyoto (WDC, Kyoto). From this examination, we find that the trend of the estimated Dst is in good agreement with that of the provided Dst when the thresholds are taken into account for both the solar wind plasma density and the dawn-to-dusk solar wind electric field, as 30 \#/cc and $0.49 \mathrm{mV} / \mathrm{m}$, respectively. From the result, we can conclude that the effect of high-density plasma is important on the enhancement of geomagnetic storm as well as the effect of the other solar wind parameters, such as the interplanetary magnetic field (IMF) Bz and solar wind velocity. On the other hand, the solar source of the magnetic field of this ICME is examined. The magnetic field structure of the ICME is examined by fitting the flux rope model to the observed magnetic field and solar wind speed. The results are compared with the magnetic structure of the bases of coronal helmet streamers. From this comparison we can find that the magnetic structure of the interplanetary flux rope is in good agreement with that of the neutral line of the base of coronal helmet streamers. The result suggests that if we look for the causes of geomagnetic storm we should take into account both the plasma structure and the magnetic structure of the base of coronal helmet streamers.
\end{abstract}

Key words: Magnetic cloud, high-density plasma, geomagnetic storm.

\section{Introduction}

ICME is frequently observed in association with enhanced magnetic field and high solar wind speed. In particular, a magnetic cloud is a kind of ICME characterized with enhanced magnetic field strength, smooth magnetic field rotation and low-temperature plasma (Burlaga et al., 1981, 1982). In addition, high-density plasma is also frequently observed in association with ICME, which often yields large geomagnetic disturbances. It is well known that the dawn-to-dusk solar wind electric field plays an important role on the enhancement of geomagnetic storm. The geomagnetic effect of high-density plasma when it associates the southward $\mathrm{Bz}$ might be also important. However this has not yet been sufficiently examined.

The estimation of Dst using solar wind parameters has been widely attempted. Burton et al. (1975) proposed the empirical relationship between solar wind conditions and Dst index. Dst index is the worldwide deviation of $\mathrm{H}$ component of geomagnetic field on disturbed days from quiet values. The $\mathrm{H}$ component of the geomagnetic field at low latitude is sensitively dependent on the variations of the magnetopause current, the ring current and the tail current.

Copyright (c) The Society of Geomagnetism and Earth, Planetary and Space Sciences (SGEPSS); The Seismological Society of Japan; The Volcanological Society of Japan; The Geodetic Society of Japan; The Japanese Society for Planetary Sciences; TERRAPUB
Burton et al. (1975) introduced all of these effects of the injected ring current, the tail current, and the magnetopause current. The magnetopause current is in proportion to the square root of the solar wind dynamic pressure. Thus, they described Dst by the following formula:

$$
\begin{gathered}
* \text { Dst }=\text { Dst }-b \sqrt{P_{d y n}}+c \\
\frac{d(* \text { Dst })}{d t}=F-a(* \text { Dst }) \\
F=0, \quad E_{y}<0.5 \mathrm{mV} / \mathrm{m} \\
F=d\left(E_{y}-0.5\right), E_{y}>0.5 \mathrm{mV} / \mathrm{m}
\end{gathered}
$$

where

$$
\begin{aligned}
d & =-1.5 \times 10^{-3} \mathrm{nT} /(\mathrm{smV} / \mathrm{m}) \\
b & =0.2 \mathrm{nT} / \sqrt{\mathrm{eV} / \mathrm{cm}^{3}} \\
c & =20 \mathrm{nT} .
\end{aligned}
$$

In the above equations, $*$ Dst represents the effect to Dst due to the injected ring current. The constant $b$ is a measure of the Dst corresponding to the solar wind dynamic pressure $\left(P_{d y n}\right)$, while $c$ is a measure of the quiet-time ring current. $F[\mathrm{nT} / \mathrm{s}]$ is the ring current injection rate that depends only upon the dawn-to-dusk solar wind electric field, $E_{y}$, which is given by $V B_{z}$, where $V$ is the solar wind velocity, $B_{z}$ is the IMF vertical component in the GSM coordinate system. 
The constant $a[1 / \mathrm{s}]$ is a measure of the ring current decay, which corresponds to e-folding time of $7.7 \mathrm{~h}$ which is a constant value.

The plasma density in solar wind has generally been considered to contribute only to the intensification of the magnetopause current, which in turn brings the recovery of Dst index. However, Fenrich and Luhmann (1998) took into account the geomagnetic effect of plasma density by considering the solar wind dynamic pressure, and modified the above formula, originally provided by Burton et al. (1975). There were two points. The first point concerns the injection function $F$, which is modified to be dependent upon the solar wind dynamic pressure as well as the solar wind electric field $E_{y}$. This modification is based upon the results of the previous study conducted by Murayama (1982). He noted a better correlation between the estimated and the measured Dst when the injection function was of the form $E_{y}\left(P_{d y n}\right)^{1 / 3}$. Thus, on the basis of the result obtained by Murayama (1982), Fenrich and Luhmann (1998) proposed the following modified function $F^{\prime}[\mathrm{nT} / \mathrm{s}], F^{\prime}=d^{\prime}\left(P_{d y n}\right)^{1 / 3}\left(E_{y}-0.5\right)$, where $d^{\prime}=-1.2 \times 10^{-3} \mathrm{nT} /(\mathrm{smV} / \mathrm{m})$, which is chosen such that $d^{\prime}\left(P_{d y n}\right)^{1 / 3}$ becomes consistent with Burton et al.'s value $d$ when $P_{d y n}$ is a typical value of $2 \mathrm{nPa}$. Thus, the density has the different effect on the Dst trend, such as the enhancement and/or recovery of the Dst. The second modification is to the ring current decay parameter $a$, which corresponds to an e-folding time of 3 or $5 \mathrm{~h}$ during the main phase when the solar wind electric field $E_{y}$ is greater than $4 \mathrm{mV} / \mathrm{m}$, and for the other cases, it is constant as $7.7 \mathrm{~h}$. Using this modified formula, Fenrich and Luhmann (1998) estimated the Dst and compared it with the provided Dst, and reported that the modified formula was more suitable than Burton $e t$ al.'s original formula.

Recently, O'Brien and McPherron (2000) proposed that the injection term $(=F)$ should be $Q[\mathrm{nT} / \mathrm{h}]=-4.4\left(E_{y}-\right.$ $0.49)$, and the decay time $\tau(=1 / a)$ varies as $\tau=$ $2.40 \exp \left(9.74 /\left(4.69+E_{y}\right)\right)[\mathrm{h}]$. Their result applies to Dst for Dst $>-150 \mathrm{nT}$, which is due to the rarity of larger excursions. Maltsev and Rezhenov (2003) carried out a statistical study using the data of Dst and of the solar wind observed over a period of about 30 years from 1963 to 1990 , and derived $Q=1.05-4.00 E_{y}-V / 243$ and $\tau=15.4 /\left(1+0.326 E_{y}\right)$. These expressions for the decay time $\tau$ mean that the decay time $\tau$ depends sensitively on the solar wind electric field $E_{y}$, and both $\tau$ values are of the same order as the constant value of $7.7 \mathrm{~h}$, originally provided by Burton et al. (1975), and the values of 7.7, 5.0, and $3.0 \mathrm{~h}$ used by Fenrich and Luhmann (1998). They show a similar profile. However, there is a difference, which increases with the increase of $E_{y}$. The dynamic pressure was not included in their injection function. We interpret their result as following. Magnetic clouds associated with high density plasma have not been frequently observed. They are the minority. Therefore, the majority is associated with low density plasma. In a statistical study, the effect of the minority clearly would disappear. In this study, we focus our attention to reveal the geomagnetic effect of high-density plasma in ICME. Therefore, the ICME with high-density plasma should be examined.
Another objective is to find the solar source of the magnetic cloud, that is ICME. The magnetic field structure of the magnetic cloud greatly affects the geomagnetic disturbance. There could be a close relationship between the magnetic field structure of magnetic cloud and that of CME. From his statistical studies Hundhausen (1993) reported that the latitude distribution of CME resembles those of prominences and coronal helmet streamers but not those of sunspots, active regions and flares, suggesting a close relationship between CMEs and the large-scale magnetic structures. Marubashi (1997) analyzed 12 magnetic clouds whose magnetic filed variations are well explained by a flux rope model, and examined the relationship between the magnetic clouds and solar magnetic field. As a result, he obtained that the solar magnetic field around disappearing filaments already had a flux rope configuration at the time of the eruption. Crooker et al. (1998) proposed the CME eruption model in which CME flux rope is created in the coronal helmet streamers. By taking account their CME model, the magnetic field structure of CME is expected to reflect the magnetic structure of the base of coronal helmet streamers.

\section{Dst and Magnetic Cloud Observed during the May 2-3, 1998 Geomagnetic Storm}

We examined the magnetic disturbance during the geomagnetic storm occurred on May 2-3, 1998 and estimated Dst index variation at the time of high density plasma observed in the magnetic cloud. The data used in this section are the solar wind parameters measured by WIND and ACE, and the Dst index provided by the WDC, Kyoto.

Figure 1 shows the solar wind parameters and the Dst index during the period from May 1 to May 4, 1998. From top to bottom, the panels illustrate, respectively solar wind speed $(V)$, proton number density $(N)$, dynamic pressure $(P)$, the most probable thermal speed $\left(V_{t h}\right)$, magnetic field intensity $\left(B_{t}\right)$, magnetic field component $B_{z}$, the ratio of alpha density to proton density and the corresponding Dst index during this period. An ICME was observed from May 2 to May 3, 1998 and caused the geomagnetic disturbance with the Dst index reaching about $-100 \mathrm{nT}$.

This ICME was dealt as a magnetic cloud since it has a clear magnetic field rotation, low temperature plasma and enhanced magnetic field intensity. This is shown with vertical lines in Fig. 1. We notice two characteristics of this magnetic cloud. First, the high-density plasma with southward $B_{z}$ was observed within the magnetic cloud. The density was higher than $30 \# / c c$, which was observed for about 15 hours. In addition, Figure 1 shows a high ratio of alpha to proton density though there was a lack of data for the period from May 2 to May 3. Helium abundance enhancement is believed to be one of the signatures of the material of the solar ejecta observed in the interplanetary space (Gosling, 1990). Secondly, the southward $B_{z}$ shows a smooth magnetic field rotation from the southward to the northward almost zero. Thus, this magnetic cloud had the high-density plasma accompanied with weak almost constant southward $B_{z}$. These conditions are very suitable for examining the effect of high-density plasma on geomagnetic disturbance because the southward $B_{z}$ is very weak.

In Sections 2.1 and 2.2, we show the estimation of Dst 


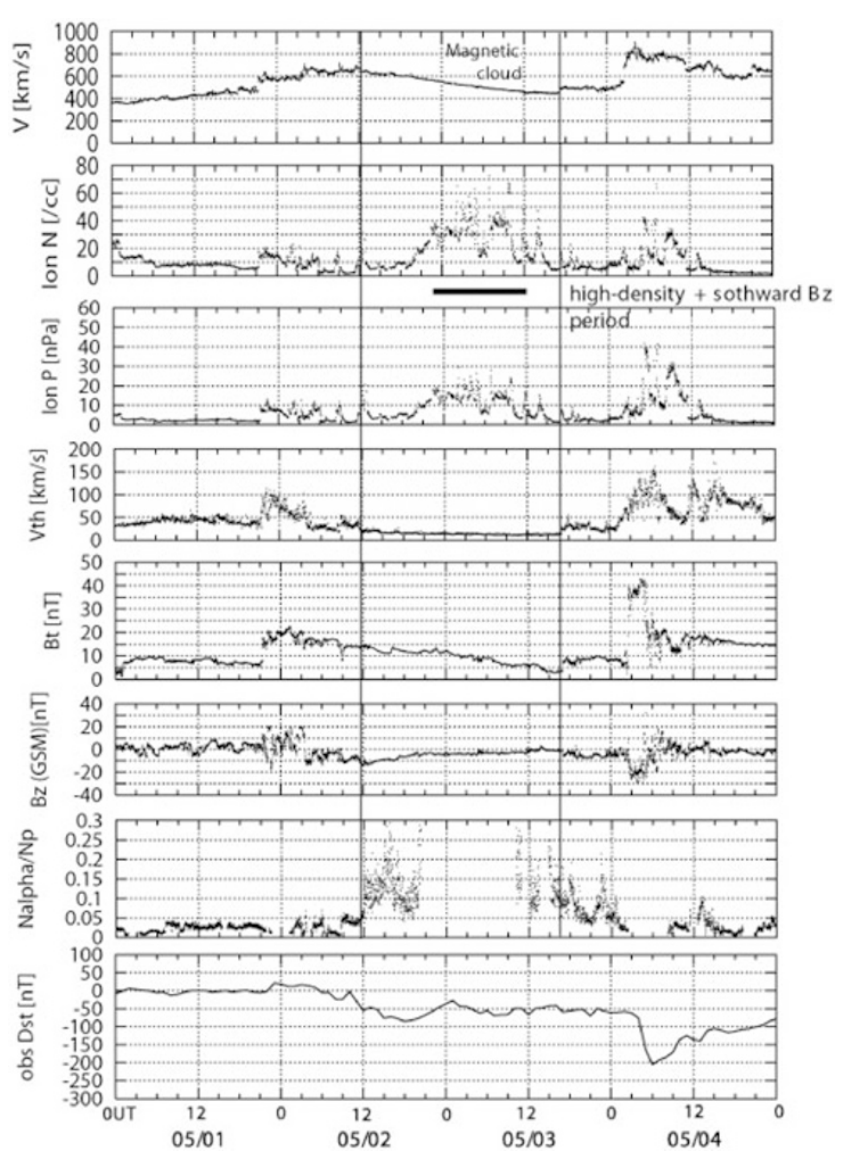

Fig. 1. The solar wind parameters observed during the period from May 1, 1998 to May 4, 1998. The top to bottom panels show characteristic variations of the solar wind parameters observed in the interplanetary space, velocity, density, dynamic pressure, thermal velocity, magnetic field, and ratio of alpha density to proton density, respectively, and the corresponding Dst index variation during this period. The high-density plasma accompanied with southward $B_{z}$ was observed on May 3 as marked with a thick solid underline.

index using the observed solar wind parameters. In Sections 3 and 4, we show the magnetic structure of the magnetic cloud, which is compared with the magnetic structure of the solar magnetic field related to the CME.

On the other hand, the interplanetary disturbance following the magnetic cloud of May 2-3 was not dealt as a magnetic cloud since the temperature was very high. On this event Burlaga et al. (2001) pointed out that the disturbance was overtaking and interacting with the preceding magnetic cloud. Thus, this was not dealt as a magnetic cloud but as "complex ejecta", which is not a flux rope and has disordered magnetic fields (Burlaga et al., 2001). The complex ejecta observed on May 4 , had a strong southward $B_{z}$ and a high-density plasma. These characteristics are not examined further in this paper.

2.1 Dst estimation for a period of the high-density plasma with southward $\mathrm{Bz}$ observed in the magnetic cloud

In order to study the effect of high-density plasma on Dst we estimate Dst by adopting different estimating formulae included the effect of solar wind plasma density. The employed formulae are those provided by Burton et al. (1975), O'Brien and McPherron (2000) and Fenrich and Luhmann
(1998), The Dst is estimated based on the observed solar wind parameters. In this estimation, we focus on the trend of Dst (enhancement or recovery) during the period of the high-density plasma observed on May 3. Figure 2 shows the estimated Dsts (solid curves) using different formulae, which are compared with the Dst index provided by the WDC, Kyoto (dotted curve) (hereinafter the provided Dst index is referred to as the provided Dst). The solar wind data used in this examination is a $10 \mathrm{~min}$ average value. The estimated Dst profiles are taken into consideration of the solar wind propagation time from WIND to the earth.

The first estimation is made on the basis of the formula provided by Burton et al. (1975), in which the injection function depends on the interplanetary electric field, Ey and the decay time is constant. Figure 2(a) shows the estimated Dst (solid curve) and the provided Dst (dotted curve). The trends of these Dst's appears similar between them for most of the period. However, there is difference during the recovery phase of the first storm on May 3, which corresponds to the period of the high-density plasma. The trend of the estimated Dst appears with a convex variation, while the provided Dst remains almost constant.

The second estimation is made on the basis of the formula provided by O'Brien and McPherron (2000). Their injection function and decay time depend on the $E_{y}$. The solid curve in Fig. 2(b) indicates the estimated Dst, which is in good agreement with the provided Dst (dotted curve) for almost the period. For the high-density period, the trend of the Dst is not coincident although the difference in the intensity almost disappears. The estimated Dst shows still a convex-type variation, while the provided Dst remains almost constant.

The third estimation was made by employing the formula provided by Fenrich and Luhmann (1998). Their injection function depends on the interplanetary dynamic pressure in addition to $E_{y}$. When $E_{y}$ is grater than $4 \mathrm{mV} / \mathrm{m}$, the decay time is adopted as 3 hour, while it is 7.7 hour for the remainder of the event. Figure 2(c) shows the estimated Dst (solid curve) and the provided Dst (dotted curve). For the highdensity period, the trend of the estimated Dst is similar to that of the provided Dst and becomes flatter than those given in Figs. 2(a) and (b). The decay time in this high-density period is 3 hours, is similar to that in Fig. 2(b). These results allow us to expect that the high-density plasma is effective on the enhancement of the geomagnetic storm. These are considered in the next section further.

\subsection{Further consideration on the effect of high-density plasma in the magnetic cloud}

In this section, we focus on the trend of Dst during the period of the high-density plasma. We improve the estimation formula on the basis of O'Brien and McPherron's (2000) formula, since it is accomplished by selecting the injection function with setting a threshold for the plasma density. We set up the threshold for the plasma density since we can expect that the effect of the high density plasma can be more clearly compared with the effect of normal density. When the plasma density $N$ was higher than $30 \# / c c$ and the interplanetary electric field $E_{y}$ was greater than $0.49 \mathrm{mV} / \mathrm{m}$, we used the injection function $F[\mathrm{nT} / \mathrm{s}]$ provided by Fenrich and Luhmann (1998). For the other period, O'Brien and 

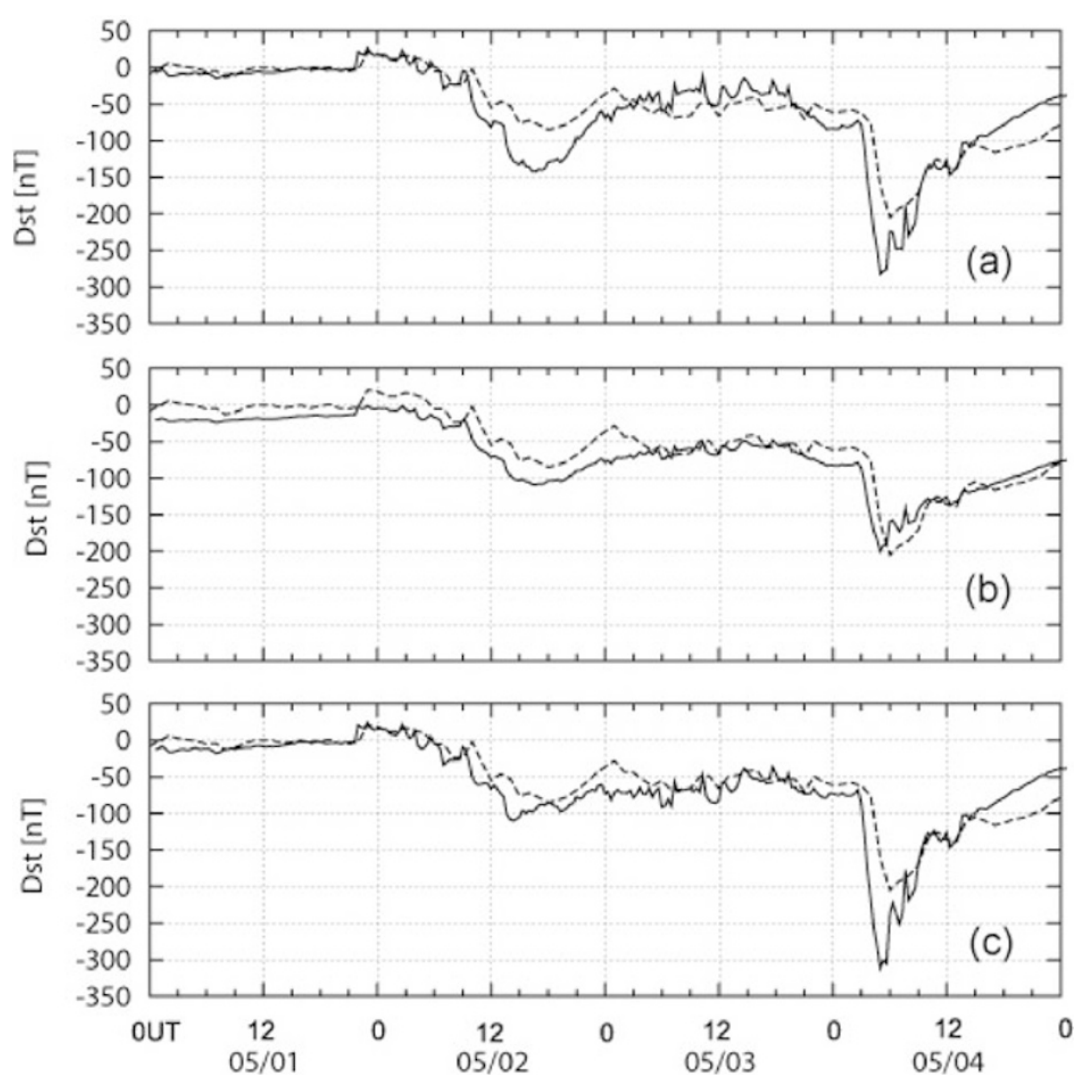

Fig. 2. The provided Dst (dashed line) and the estimated Dst (solid line) from May 1, 1998 to May 4, 1998. The estimation in Fig. 2(a) is obtained using the formula provided by Burton et al. (1975). Figure 2(b) is based on the formula provided by O'Brien and McPherron (2000). The estimation in Fig. 2(c) is based on the formula provided by Fenrich and Luhmann (1998).

McPherron's (2000) injection function $F$ [nT/s] was used. Here we used the threshold value of the interplanetary electric field $E_{y}$ as $0.49 \mathrm{mV} / \mathrm{m}$ in place of $0.5 \mathrm{mV} / \mathrm{m}$, which is used in the original injection function provided by Fenrich and Luhmann (1998). It is changed in order to relate it to the O'Brien and McPherron's (2000) injection function. Thus, the improved formula is as follows.

$$
\begin{aligned}
& * \mathrm{Dst}=\mathrm{Dst}-7.26 \sqrt{P_{d y n}}+11 \\
& \frac{d(* \mathrm{Dst})}{d t}=F-a(* \mathrm{Dst}) \\
& 1 / a=2.4 \exp \left(9.74 /\left(4.69+E_{y}\right)\right) \times 3600[s] \\
& E_{y}=\left|V B_{z}\right|, B_{z}<0 \\
& E_{y}=0, \quad B_{z} \geq 0
\end{aligned}
$$

$F=0 \quad[\mathrm{nT} / \mathrm{s}]$,

$$
E_{y} \leq 0.49
$$

$F=-1.2 \times 10^{-3}\left(P_{d y n}\right)^{1 / 3}\left(E_{y}-0.49\right), E_{y}>0.49, N>30$

$F=-1.2 \times 10^{-3}\left(E_{y}-0.49\right)$,

$E_{y}>0.49, N \leq 30$

The result is shown in Fig. 3. For the high-density period on May 3, the trend of the estimated Dst becomes almost constant. By introducing the modified formula, the change is small compared with the deviation of the Dst.

In order to see the change more clearly we calculate the first derivative of the provided and estimated Dsts given in Figs. 2(a), (b), and 3. The derivative results are shown in Fig. 4(a), (b) and (c), respectively. The horizontal bar in the figure indicates the high-density plasma period of the magnetic cloud. In order to compare the trend of the provided Dst in detail the estimation of Dst is set to the value at the time when the provided Dst was minimum on May 4. This set is effective since at the minimum value of the provided Dst, the variation of the Dst is largest in this period.

Figure 4(a) shows the comparison of the first derivatives of the Dst estimated by Burton et al. (1975) original formula (solid line) and of the provided Dst (dashed line) during the period from 18 UT May 2 to 18 UT May 3. They show positive and negative values, respectively. It is clearly understood from this figure that the trend for the high-density period is different each other.

Figure 4(b) shows the comparison of the first derivatives of the Dst estimated by O'Brien and McPherron's (2000) original formulae (solid line) and of the provided Dst (dashed line) during the same period of Fig. 4(a). They show positive and negative values, respectively. The decay time depending on the $E_{y}$ doesn't clarify the difference of the trend.

On the other hand, in Fig. 4(c), the derivative values are presented for the Dst estimated by the modified formula for Fig. 3 (solid line) and for the provided Dst (dashed line) for the same period of Figs. 4(a) and (b). They show negative values during the high-density period. Therefore, the trends are almost same each other. From these examinations, we can conclude that the high density plasma accompanying southward $B_{z}$ contributes effectively to the enhancement of the geomagnetic disturbances. 


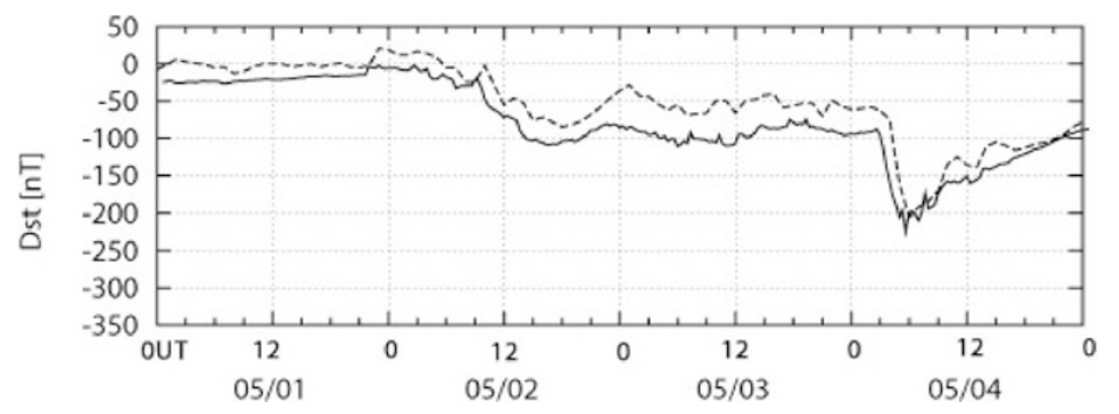

Fig. 3. The solid line shows the improved estimation using Fenrich and Luhmann's injection function for the period of the high-density plasma, with setting the threshold for both the plasma density and the electric field. In the remaining period, O'Brien and McPherron's injection function is applied. The dashed line shows the provided Dst.

\section{Magnetic Structure of the Magnetic Cloud}

In this section, we examine the magnetic structure of the magnetic cloud observed on May 2-3. We used a constantalpha force free cylindrical model including a self-similar expansion (Burlaga, 1988; Farrugia et al., 1992; Marubashi, 1997), and carried out the model fitting for the observed solar wind data during the period of the magnetic cloud using the nonlinear least-squares fitting technique developed by Marubashi (1997). In this model, the magnetic field, $\vec{B}$, the flux rope radius, $R$, and the expansion velocity, $\vec{v}$, are expressed in cylindrical coordinates as follows:

$$
\begin{gathered}
\vec{B}=B_{\phi} \overrightarrow{e_{\phi}}+B_{Z} \overrightarrow{e_{Z}} \\
B_{\phi}=s B_{0} J_{1}(a r) /(1+\varepsilon t) \\
B_{Z}=B_{0} J_{0}(a r) /(1+\varepsilon t)^{2} \\
B_{r}=0 \\
R=R_{0}(1+\varepsilon t) \\
\vec{v}=v_{r} \overrightarrow{e_{r}} \\
v_{r}=\varepsilon r /(\varepsilon t+1) \quad(r \leq R)
\end{gathered}
$$

where $B_{\phi}$ is the component of the magnetic field in azimuthal direction, $B_{Z}$ is the component of the magnetic field along the axis of the flux rope, and $B_{r}$ is the radial component of the magnetic field in the cylindrical coordinate. Supposing $R_{0}$ to be the radius of a flux rope at the first encounter with the satellite, $B_{0}$ is the magnetic field intensity at the flux rope axis, $t$ is the time after the first encounter, $v_{r}$ is the expansion speed at time $t$, and $\varepsilon$ is the expansion rate. $J_{0}$ and $J_{1}$ are Bessel functions of the first kind of order 0 and 1. $a$ is such that $a R$ gives the first zero of $J_{0}$. The handedness of the magnetic field in the flux rope is represented by $s=1$ or $s=-1$ for parallel (right-handed) and anti parallel (left-handed), respectively. Impact parameter $p$ is a unit distance measured along the vector product, $\overrightarrow{e_{x}} \times \overrightarrow{e_{Z}}$ (for $\mathrm{X}$-component in the GSE coordinate and for Z-component in the cylindrical coordinate, respectively), and it can take either a positive or negative value. For this model fitting, we need the numerical data about the observed interplanetary magnetic field and solar wind velocity during the period when the magnetic cloud passed through the satellite.

The result of the fitting is shown in Fig. 5. The parameters given in Fig. 5 from top to bottom are the solar wind velocity, the magnetic field intensity, and three components
Table 1. The flux rope parameters calculated by the flux rope fitting.

\begin{tabular}{lc}
\hline$R_{0}$ & $0.2 \mathrm{AU}$ \\
\hline$p$ & 0.9 \\
\hline LAT & $-73 \mathrm{deg}$. \\
\hline LON & $164 \mathrm{deg}$. \\
\hline$s$ & 1 \\
\hline
\end{tabular}

of the magnetic field, $B_{x}, B_{y}$, and $B_{z}$ in GSE coordinates, respectively. The solid line shows the fitted curve. The circles are the data used for the fitting, which were a one houraverage value based on the observation in the interplanetary space. The solar wind velocity decreased monotonically during this flux rope. This profile indicates the expansion of the flux rope since the sum of the expansion velocity and the bulk velocity of the flux rope is the observed solar wind velocity. Table 1 shows the flux rope parameters for the structure. From top to bottom, the radius of the flux rope at the time of the first encounter, $R_{0}=0.2 \mathrm{AU}$, the impact parameter $p=0.9$, the latitude angle of the axial magnetic field LAT $=-73 \mathrm{deg}$., the longitude angle of the axial magnetic field LON = $164 \mathrm{deg}$. and the handedness of the magnetic field $s=1$, means the right-handedness. Thus, the axis of this flux rope almost points to $-z$-axis of GSE coordinates. Thus, the result suggests that the spacecraft passed near the limb of this flux rope, where the variation of the latitude angle of the magnetic filed was considered to be small with the weak $B_{z}$.

\section{Relationship between the Magnetic Cloud Ob- served on May 2-3, 1998 and the CME}

The magnetic cloud observed on May 2-3, 1998 was associated with the halo-CME observed on the LASCO C2 coronagraph on SOHO starting at 1658 UT on April 29, 1998. This was based on the reasoning that we can expect that the solar source of this magnetic cloud should be seen during the time window of about one day before and after the time calculated under the assumption that the magnetic cloud travels constantly from the sun to the earth with the bulk velocity of this interplanetary flux rope. The direction of CME, away from the earth or toward the earth, cannot be determined only from the observation of the coronagraph. CME has been frequently observed in association with fila- 

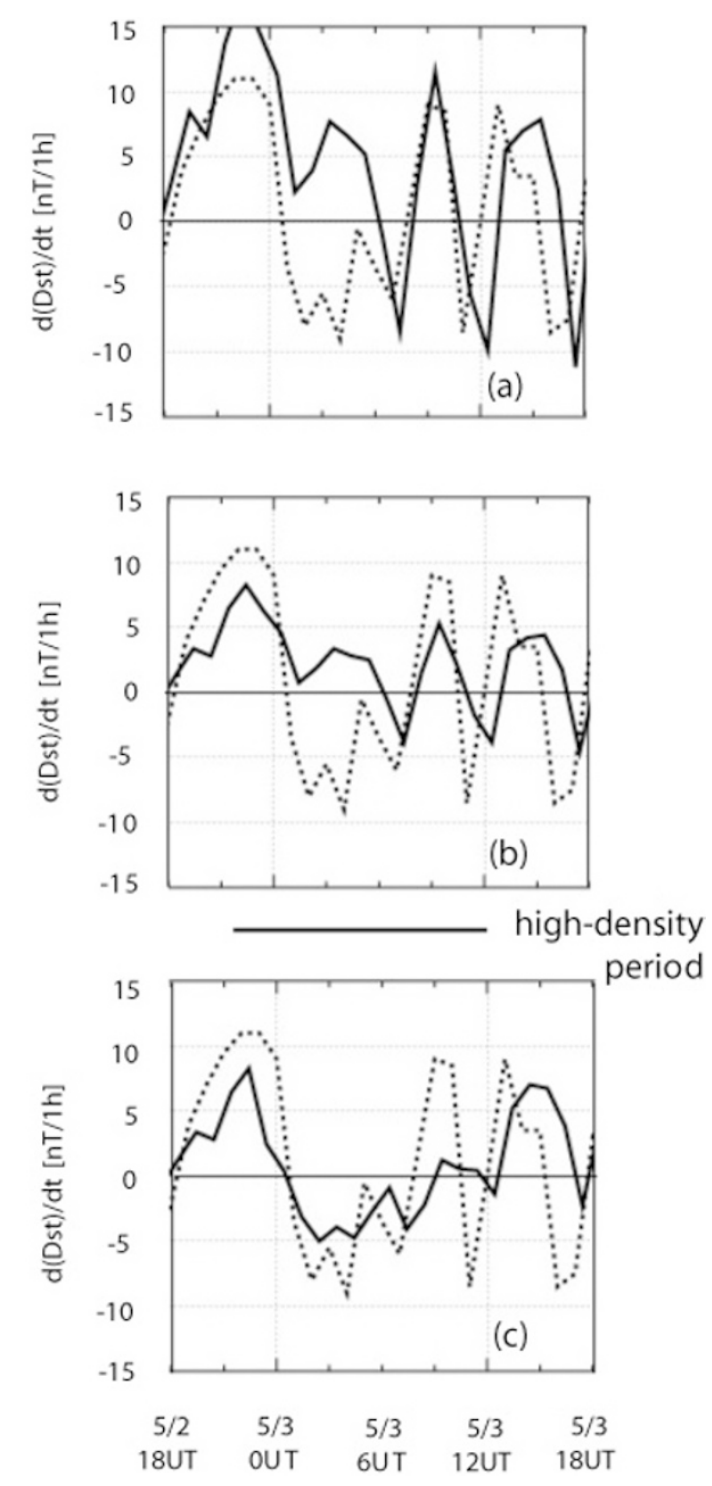

Fig. 4. The first derivative values of the provided Dst (dashed line) and the estimated Dst (solid line). The solid line in Figs. 4(a) and (b) show the first derivative values of the Dst estimated by the formulae provided by Burton et al. (1975) and by O'Brien and McPherron (2000), respectively. Opposite trends can be seen during the high-density period on May 3. The solid line in Fig. 4(c) shows the first derivative values of the Dst estimated by considering the effect of the dynamic pressure in the injection function. The first derivative values of the provided Dst and of the estimated Dst show the same trend.

ment eruptions (e.g. Burlaga et al., 2001). Since this CME was a halo-type CME associated with the following observed phenomena, such as M-class flare, filament disappearance at NOAA 8210 (Haimin et al., 2000; Moon et al., 2002) and dimming (Thompson et al., 2000), we can expect that this CME propagated toward the earth rather than away from it. Thus, it is reasonable to conclude that this CME was related to the magnetic cloud although there was no evidence that the plasma material of this CME was the same as that observed in the magnetic cloud.

We examined the relationship between the magnetic structure of the interplanetary flux rope and the related CME by taking account of the CME model proposed by Crooker et al. (1998). In their model, the CME flux rope

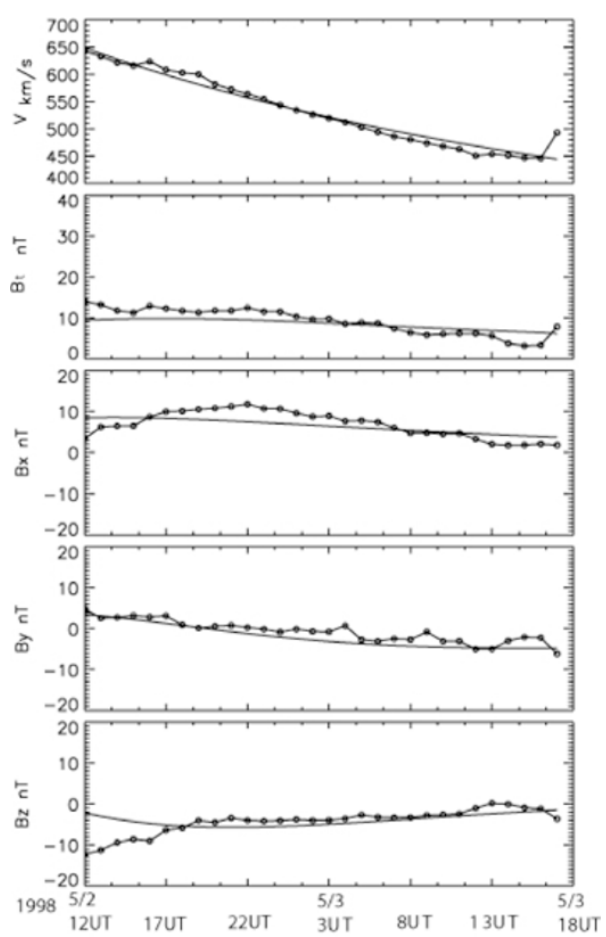

Fig. 5. The result of flux rope fitting to the magnetic cloud observed from 12 UT May 2 to 17 UT May 3, 1998. From top to bottom the panels are the solar wind parameters, i.e., the solar wind speed, magnetic field intensity, and three components of the magnetic field, $B_{x}, B_{y}$ and $B_{z}$ in GSE coordinates, respectively. The solid line shows the fitted curve. The circles indicate the observed values by WIND.

is constructed in the coronal helmet streamers and there is a close relationship between the structure of the magnetic field constructing the CME flux rope and the coronal helmet streamers. Furthermore, when the CME flux rope could propagate through the interplanetary space without strong interactions, the magnetic structure of the CME flux rope, i.e., that of the base of the coronal helmet streamers, is expected to agree with that of the interplanetary flux rope. Thus, we examined the base of coronal helmet streamers for this CME and compared the magnetic structure with that of the interplanetary flux rope.

This was done based on the computed source surface field map at 2.5 solar radii provided by the Wilcox Solar Observatory at Stanford University. The solar magnetic field when the CME occurred, is shown in Fig. 6(a). The vertical line indicates the central meridian on April 29 at the occurrence of the CME. The solid and dashed lines respectively show the equi contour of the field intensity for positive and negative polarities, and the boundary line between the two polarities shows the neutral line. The neutral line indicates the location of the base of the Heliospheric current sheet (HCS) (Zhao and Hoeksema 1996). The HCS forms where solar wind flows carrying magnetic fields of opposite polarity converge over magnetically closed regions of the corona, i.e., coronal helmet streamers. Thus, we estimated the base of the coronal helmet streamers from the neutral line. Figure 6(a) indicates that the region of the filament disappearance, i.e., NOAA 8210 marked by the solid point, was located under the base of the coronal helmet streamers. The neutral line on April 29 in this map was in the north- 

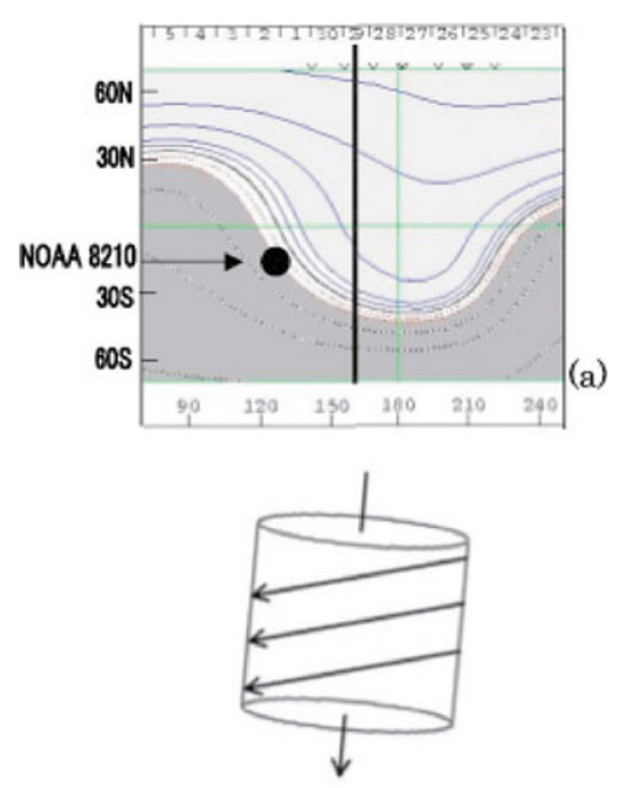

(b)

Fig. 6. The upper panel (a) shows the computed source surface field map at 2.5 solar radii (Rs) provided by the Wilcox Solar Observatory at Stanford University. The vertical line and the solid circle indicate the central meridian of the day and the location of NOAA 8210 , respectively. The solid and dashed lines respectively show the equi- contour of field intensity for positive and negative polarities. The boundary line between two polarities shows the neutral line. The bottom panel (b) shows the magnetic structure of the interplanetary flux rope for the magnetic cloud observed on May 2-3 estimated from the flux rope fitting. This is projected onto the yz-plane.

south direction, and the magnetic polarity was positive in the side of the central meridian of the day and negative in the side of the limb.

Figure 6(b) shows the interplanetary flux rope obtained by the flux rope fitting. The axis of the flux rope points almost to $-\mathrm{z}$ axis in GSE coordinates. The handedness is right-handed. Those are compared with the observations of the direction of the neutral line and of the magnetic polarity around the neutral line on April 29, given in Fig. 6(a). As a result, we can confirm that the magnetic structure of the interplanetary flux rope seems to be very consistent with that of the base of the coronal helmet streamers corresponding to the $\mathrm{CME}$.

\section{Summary and Discussion}

Our objective in this paper is to clarify the geomagnetic effect of high density plasma in the ICME, i.e., how degree the solar wind density affects the recovery or the enhancement of Dst index variations. In this paper we focused our attention to the trend of Dst index variation rather than its absolute magnitude of the Dst index variation. The trend of the provided Dst during the period of the high-density plasma remained almost constant. However, the estimations based on the formulae provided by Burton et al. (1975) and O'Brien and McPherron (2000) showed the trends with the convex-type variation. Thus, the injection functions introduced in the formulae provided by Burton et al. (1975) and O'Brien and McPherron (2000), which depend only on Ey term couldn't explain the difference of between the trends of the estimated and the provided Dsts. The difference wasn't clear by introducing the decay time.

In order to reduce the difference we adopted another injection function developed by Fenrich and Luhmann (1998), which includes the term of dynamic pressure (plasma density). Here, we set afresh a threshold for the plasma density $N$, where the injection function was used when the plasma density $N$ was larger than $30 \# / c c$. While the plasma density $N$ was less than $30 \# / c c$, O'Brien and McPherron's (2000) injection function was applied. The result shows that the trend of the estimated Dst is in good agreement with that of the provided Dst as shown in Figs. 3 and 4(c). From this result, we can conclude that the highdensity plasma with southward $B_{z}$ included in the ICME can contribute to the enhancement of the geomagnetic disturbance.

The density threshold, $N=30 \# / \mathrm{cc}$, isn't strict for the distinction between high and normal density. In this event, the effect of the high-density plasma clearly appeared on Dst enhancement as shown in Figs. 4(a), (b) and (c), when three situations, high-density plasma, weak southward $B_{z}$ and small variation southward $B_{z}$, are coincident. It is not always that the Dst enhancement appears when the highdensity plasma is observed with southward $B_{z}$. The formula used for the estimation of Dst index variation indicates that the trend of the estimated Dst is controlled by the amount of the contribution of the electric filed and that of the dynamic pressure. The former contributes to the enhancement of Dst, while the latter to the enhancement and/or recovery of Dst. This is an important point to be examined further.

Next we examined what is the source of the observed high-density plasma discussed above? There might be two candidates as follows;. 1) The high-density plasma originated from the sun. 2) The high-density plasma produced in interplanetary space due to the compression between fast and slow solar winds (Crooker, 2000). From Fig. 1, the interplanetary disturbance observed on May 2-3 showed signatures of magnetic could. In addition, it showed the highalpha density within it. These characteristics are consistent with those of interplanetary counterparts of CME (Gosling, 1990). Thus, we can conclude that the high-density plasma within the magnetic cloud is considered to be originated from the sun.

On the other hand, for the complex ejecta observed on May 4, the origin of the high-density plasma isn't obvious since it has been already constructed through the complex interactions. Burlaga et al. (2001) suggested that the complex ejecta observed from May 4, 1998 were accompanied with at least three full-halo CMEs and three partial-halo CMEs since the large fluctuations in temperature and solar wind velocity were observed within the complex ejecta. Furthermore, from the statistical study about the fast ejecta observed from 1998 to 1999, Burlaga et al. (2001) suggested that the majority of magnetic clouds are associated with a single solar source but nearly all of the complex ejecta have a multiple source. Generally, CME frequently occurs during the solar maximum. Thus, the compression in interplanetary space frequently might occur. That can yield 
high-density plasma.

We examined the relationship between the magnetic structure of the magnetic cloud observed from May 2 to May 3, 1998 and that of the corresponding CME. As a result of the comparison of Figs. 6 (a) and (b), the magnetic polarity and inclination of the interplanetary flux rope were found to be in agreement with those of the base of the coronal helmet streamer estimated from the neutral line on the source surface map. Hundhausen (1993) reported the close relationship between the CME and the large-scale magnetic structures, such as prominences and coronal helmet streamers, rather than small-scale magnetic structures such as sunspots, active regions and flares. Thus, the result obtained by Hundhausen (1993) supports the idea that the magnetic structure of the magnetic cloud is related to that of the coronal helmet streamers. Crooker et al. (1998) proposed the CME model, in which the base of the CME flux rope is in that of the coronal helmet streamers. The CME corresponding to the magnetic cloud observed on May 2-3, 1998 was associated with the filament disappearance under the coronal helmet streamers though not all CME necessarily arise from the region of the main streamer belts. In addition, Zhao and Webb (2003) found that most of the central position of front-side full-halo CME from 1997 to 2000, including the event studied in this paper, were located under bipolar coronal streamer belts on the sun and suggested that bipolar coronal helmet streamers might be the source regions of CME. Thus, our conclusion seems to be reasonable.

The profile of the provided Dst in Fig. 1 is similar to that of the southward $B_{z}$. This indicates that the effect of the southward $\mathrm{Bz}$ on the Dst might be important. Our results, that is, the magnetic structure of the coronal helmet streamers is related to that of the magnetic cloud, and the magnetic structure of the magnetic cloud is also intimately related to the Dst profile.

Thus, we can conclude that the magnetic structure of the coronal helmet streamers might be important for considering the cause of geomagnetic disturbance, as observed during the May 2-3 1998 storm. This conclusion suggests the importance of the observation of CMEs for the prediction of the space weather.

Acknowledgments. We appreciate the WIND MFI team for providing the WIND MFI data (http://lepmfi.gsfc.nasa.gov/), the WIND SWE team for providing the WIND SWE data, the ACE SWEPAM team and the ACE SCIENCE CENTER for providing ACE SWEPAM Level 2 data, CDAWeb (http://cdaweb.gsfc.nasa. gov/) for providing the WIND SWE data and the ACE SWEPAM Level 2 data, the World Data Center for Geomagnetism, Kyoto for providing the Dst index, the Wilcox Solar Observatory at Stanford University for providing the computed source surface field map (http://quake.stanford.edu/ wwso/coronal.html), and the SOHO LASCO team for providing the SOHO LASCO CME catalog (http://cdaw.gsfc.nasa.gov/CME_list/).The data and calculations for the computed source surface field map are from the Wilcox Solar Observatory at Stanford University. This CME cata$\log$ is generated and maintained by NASA and The Catholic Uni- versity of America in cooperation with the Naval Research Laboratory. SOHO is a project of international cooperation between ESA and NASA.

\section{References}

Burlaga, L. F., Magnetic cloud and force-free fields with constant alpha, $J$. Geophys. Res., 93, 7217-7224, 1988.

Burlaga, L. F., E. Sittler, F. Mariani, and R. Schwenn, Magnetic loop behind an interplanetary shock: Voyager, Helios and IMP8 observations, J. Geophys. Res., 86, 6673-6684, 1981.

Burlaga, L. F., L. Klein, N. R. Sheeley, Jr., D. J. Michels, R. A. Howard, M. J. Koomen, R. Schwenn, and H. Rosenbauer, A magnetic cloud and a coronal mass ejection, Geophys. Res. Lett., 9, 1317-1320, 1982.

Burlaga, L. F., R. M. Skoug, C. W. Smith, D. F. Webb, T. H. Zurbuchen, and A. Reinard, Fast ejecta during the ascending phase of solar cycle 23: ACE observations, 1998-1999, J. Geophys. Res., 106, 20,957-20,977, 2001

Burton, R. K., R. L. McPherron, and C. T. Russell, An empirical relationship between interplanetary conditions and Dst, J. Geophys. Res., 80 , 4204-4214, 1975.

Crooker, N. U., Solar and Heliospheric geoeffective disturbances, J. Atmos. Solar-Terr. Phys., 62, 1071-1085, 2000.

Crooker, N. U., J. T. Gosling, and S. W. Kahler, Magnetic cloud at sector boundaries, J. Geophys. Res., 103, 301-306, 1998.

Farrugia, C. J., L. F. Burlaga, V. A. Osherovich, and R. P. Lepping, A comparative of dynamically expanding force-free, constant-alpha magnetic configurations with applications to magnetic clouds, in Solar Wind Seven, edited by E. Marsch and R. Schwenn, COSPAR, vol. 3, 711 pp, Pergamon, New York, 1992.

Fenrich, F. R. and J. G. Luhmann, Geomagnetic response to magnetic clouds of different polarity, Geophys. Res. Lett., 25, 2999-3002, 1998.

Gosling, J. T., Coronal mass ejections and magnetic flux ropes in interplanetary space, in Physics of Magnetic flux ropes, Geophys. Monogr. Ser., vol. 58, edited by C. T. Russell, E. R. Priest, and L. C. Lee, 685 pp., AGU, Washington, D.C., 1990.

Haimin, W., P. R. Goode, C. Denker, G. Yang, V. Yurchishin, N. Nitta, J. B. Gurman, C. St. Cyr, and A. G. Kosovichev, Comparison of the 1998 April 29 M6.8 and 1998 November 5 M8.4 flares, ApJ., 536, 971-981, 2000.

Hundhausen, A. J., Sizes and locations of coronal mass ejections: SMM observations from 1980 and 1984-1989, J. Geophys. Res., 98, 13,177$13,200,1993$

Maltsev, Yu. P. and B. V. Rezhenov, Relation of the Dst index to soalr wind parameters, Int. J. Geomagn. Aeron., 4, 1-9, 2003.

Marubashi, K., Interplanetary magnetic flux ropes and solar filaments, in Coronal Mass Ejections, Geophys. Monogr. Ser., vol. 99, edited by N. Crooker, J. A. Joselyn, and J. Feynman, 299 pp, AGU, Washington, D.C., 1997.

Moon, Y.-J., J. Chae, H. Wang, G. S. Choe, and Y. D. Park, Impulsive variations of the magnetic helicity change rate associated with eruptive flares, ApJ., 580, 528-537, 2002.

Murayama, T., Coupling function between solar wind parameters and geomagnetic indices, Rev. Geophys. Space. Phys., 20, 623-629, 1982.

O'Brien T. P. and R. L. McPherron, An empirical phase space analysis of ring current dynamics: Solar wind control of injection and decay, $J$. Geophys. Res., 105, 7707-7719, 2000.

Thompson, B. J., E. W. Cliver, N. Nitta, C. Delannee, and J.-P. Delaboudiniere, Coronal dimmings and energetic CMEs in April-May 1998, Geophys. Res. Lett., 27, 1431-1434, 2000.

Zhao, X. P. and J. T. Hoeksema, Effect of coronal mass ejections on structure of the heliospheric current sheet, J. Geophys. Res., 101, 48254834, 1996.

Zhao, X. P. and D. F. Webb, Source regions and storm effectiveness of frontside full halo coronal mass ejections, J. Geophys. Res., 108, SSH41-SSH-4-11, 2003.

H. Adachi (e-mail: adachi@ea.u-tokai.ac.jp), T. Sakurai, and K. Marubashi 